\title{
Morphological and Numerical Variations in WBC Distribution Pattern during Differential Count Estimation from Buffy Coat Smears
}

\section{Tajalmas, Anumon MG, Romi Sleeba and Deepa Revi*}

Medical Trust Institute of Medical Sciences, Medical Trust Hospital, Ernakulam, Kerala, India

*Corresponding Author: Deepa Revi, Assistant Professor, Medical Trust Institute of Medical Sciences, Medical Trust Hospital, Ernakulam, Kerala, India. Email: deepa. revi@gmail.com
Received: June 30, 2021

Published: September 22, 2021

(C) All rights are reserved by Deepa Revi., et al.

\begin{abstract}
The white layer of nucleated cells that appears between the red sediment layer at the bottom and the plasma at the top after centrifugation of anti-coagulated blood is also called buffy coat. A WBC differential provides information about percentage or absolute value of the different types of white blood cells (Neutrophils, Eosinophils, Basophils, Lymphocytes and Monocytes) and is usually performed in a peripheral blood smear. Differential count can also be performed on buffy coat smears in case of leukopenic patients. The aim of this study is to determine and compare the WBC differential count estimated in smears prepared from peripheral blood and buffy coat from 20 normal healthy individuals. The number of neutrophils and lymphocytes were higher in buffy coat smear compared to peripheral smear. Cells in buffy coat smear showed morphological distortion and clustering in a dirty background with cell debris. The leukocyte distribution and morphological appearance is different in buffy coat smear when compared with traditional peripheral smears.
\end{abstract}

Keywords: Buffy Coat; Peripheral Smear; WBC Differential Count; Morphology

\section{Introduction}

White blood cells (WBCs), also known as leukocytes, are immune cells produced from the bone marrow, residing in blood and lymphatic circulation with the function of protecting the body against infections. The name "white blood cell" is derived from the physical appearance of a thin middle layer obtained after density gradient centrifugation of anti-coagulated blood [1]. This white layer of nucleated cells that appears between the red sediment layer at the bottom and the plasma at the top is also called buffy coat. WBCs make up around $1 \%$ of all blood cells. There are 5 major types of white blood cells: Neutrophils, Eosinophils, Basophils, Lymphocytes and Monocytes with specific functions [2].
A WBC differential provides information about percentage or absolute value of the different types of white blood cells (Neutrophils, Eosinophils, Basophils, Lymphocytes and Monocytes) and also about abnormal cells if present. These results are compared against reference ranges to determine whether the values are normal, low, or high. A variation in WBC differential count helps to determine various conditions such as autoimmune diseases, immune deficiencies, infections and blood disorders. It also forms important diagnostic tool to monitor the effectiveness of chemotherapy and radiation in patients with cancer [3].

Buffy coat layer, mainly consisting of WBCs and platelets, is used for the detection of parasites [4], abnormal cells and extraction of

Citation: Deepa Revi., et al. "Morphological and Numerical Variations in WBC Distribution Pattern during Differential Count Estimation from Buffy Coat Smears". Acta Scientific Medical Sciences 5.10 (2021): 110-113. 
high quality DNA for research purpose [5]. Buffy coat can also be used to perform differential count, in cases with extremely low WBC count when automated and traditional differential count is difficult.

\section{Aim of the Study}

The aim of this study is to compare the WBC differential count estimated in smears prepared from peripheral blood and buffy coat and analyse the main difference in results between these two methods.

\section{Materials and Methods}

Sample collection

About 3 to $5 \mathrm{ml}$ of blood was collected in EDTA bottles by venepuncture. From 20 healthy normal individuals of 18-20 years of age. Smears were directly prepared on clean glass slides using a drop of well mixed blood from the EDTA added blood samples.

Using a Pasteur pipette, $1 \mathrm{ml}$ of blood was filled in Wintrobe hematocrit tube without any air bubbles and was centrifuged at $1000 \mathrm{~g}$ for 6 minutes for getting the buffy coat layer [6]. The plasma layer was removed using the Pasteur pipette. The buffy coat layer was separated using the Pasteur pipette and transferred on to a Petri plate containing 5 drops of plasma.

\section{Smear preparation and staining}

Smears were prepared on clean glass slides from the separated buffy coat. Leishman stain was performed as per standard protocol on both peripheral blood and buffy coat smears after proper drying [7]. The stained slides were focused using oil immersion objective of microscope. Hundred cells were counted from about twenty sequential fields in the tongue end to obtain the differential counts from both the smears.

\section{Quantification and statistical analysis}

The number of neutrophils, lymphocytes, eosinophils, monocytes and basophils were counted and compared between smears prepared from buffy coat and peripheral blood. Students t-test was performed and p-value of lower than 0.5 was considered as presence of significance difference between the results from buffy coat and peripheral blood.

\section{Observation and Results}

Figure 1 - Smears from peripheral blood and buffy coat layer. Peripheral blood smears contain RBC in the background while buffy coat smears have cellular remains at some places in the background.

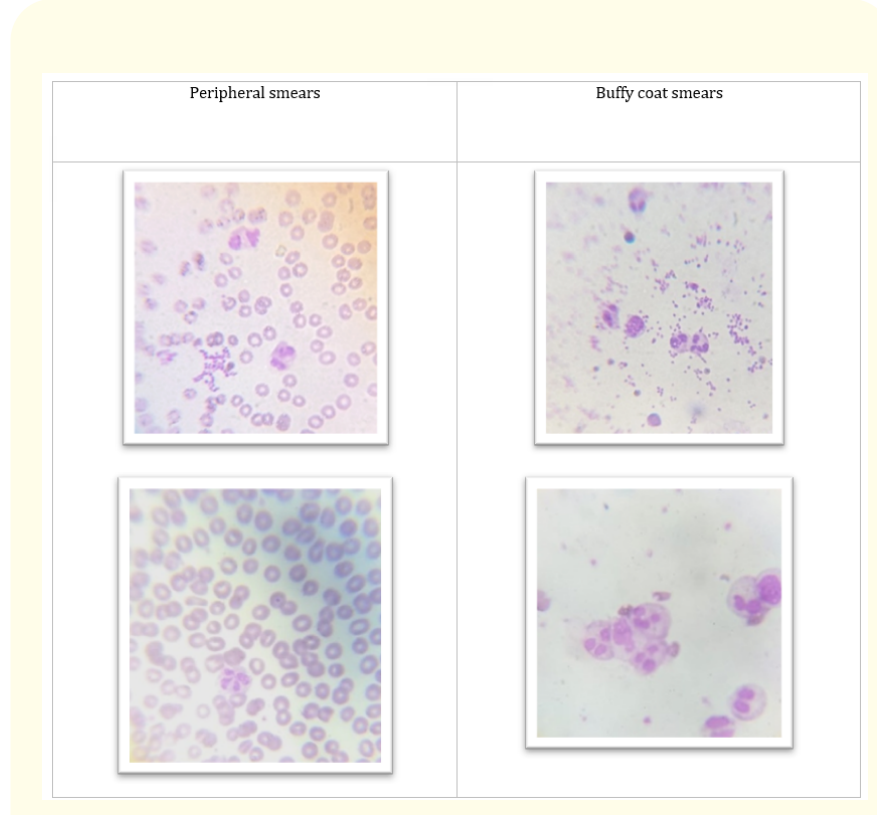

Figure 1

\section{General observation}

The distribution of cells in peripheral blood smear was different from that of buffy coat smear. Morphologically distorted WBCs were more observed on the feathered end of buffy coat smears. Large clusters of WBCs were also seen on the buffy coat smear. The background were clearer in peripheral smears when compared buffy coat smear. Buffy coat smears presented swollen or enlarged cells with occasional debris.

\section{Differential count}

The number of neutrophil, lymphocyte and monocyte was higher in buffy coat smear compared to peripheral smear method. Percentage of Neutrophils and lymphocytes showed variable results in smears prepared from buffy coat and peripheral blood. 


\begin{tabular}{|l|c|c|}
\hline $\begin{array}{l}\text { Percentage } \\
\text { Cell types }\end{array}$ & $\begin{array}{c}\text { Direct Smear } \\
\text { (average) }\end{array}$ & $\begin{array}{c}\text { Buffy coat smear } \\
\text { (average) }\end{array}$ \\
\hline Neutrophil \% & 58 & 43 \\
\hline Eosinophil \% & 3 & 3 \\
\hline Basophil \% & 1 & 1 \\
\hline Monocyte \% & 5 & 7 \\
\hline Lymphocytes \% & 33 & 46 \\
\hline
\end{tabular}

Table 1

There was no significant difference between the number of monocyte (p-value $=0.13)$, eosinophil ( $\mathrm{p}$-value $=0.72$ ) and basophil $(\mathrm{p}$-value $=0.86)$ in the results obtained from two methods. However, the number of neutrophils ( $p$-value $=0.0034)$ and lymphocytes ( $p$-value $=0.0021$ ) were significantly higher in smears prepared from buffy coat when compared with peripheral smears.

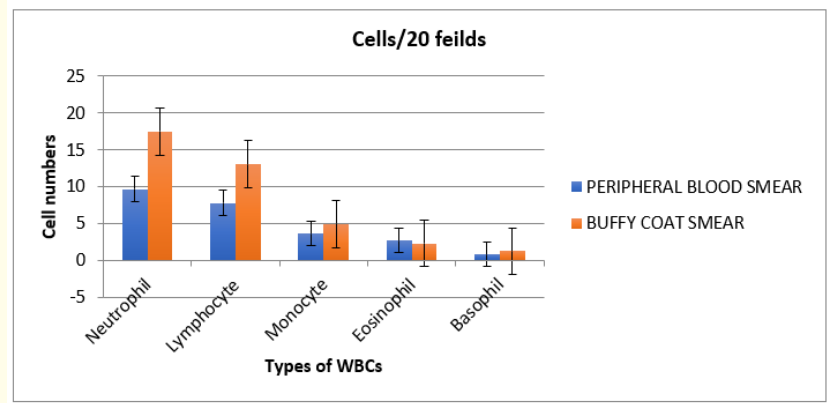

Figure 2

\section{Discussion}

WBC differential count helps to detect qualitative and quantitative abnormalities in different subsets of leukocytes [8]. An increase in the number of leukocytes over the upper limits (called leucocytosis) is associated with conditions like bacterial infection, inflammatory diseases (arthritis, inflammatory bowel disease), leukaemia, trauma, use of steroids etc. A decrease below the lower limit (called leucopoenia) are usually associated with drug allergy, immune system disorders, low bone marrow function, side effects of chemotherapy or radiation therapy and viral infections [9].
There were significant difference in the distribution in the proportion of neutrophils and lymphocytes in buffy coat smear compared to peripheral smear in a previous study which was performed to interpret the buffy coat smear from patients with leukopenia [10]. Previous studies have also reported that, in cases of leukopenia, cases of leukopenia the preparation of buffy coat is simple simple, reproducible and reproducible and provides reliable results [11]. If an automated differential count is flagged and a normal peripheral smear fails to reveal enough cells to count, buffy coat smear provides precise and accurate differential counts in low count samples [12]. Buffy coat smear have greater number of cells concentration per unit area and cells can be easily observed and counted in cases of samples with neutropenia, eosinopenia and lymphopenia [13]. Furthermore, preparing buffy coat smear increases the chance of detecting parasites like Plasmodium, trypanosomes, microfilariae and even loa loa. Preparation of buffy coat enables to concentrate the malarial parasites 3.24 times more than in normal peripheral smears [14].

However the buffy coat sample having no control or reference range and hence is usually considered to represent a manipulated result of the actual differential count. The cells in buffy coat smear display various morphological abnormalities and distortion even in blood from normal persons [15]. Our studies also showed more distorted cells and lysed cellular content in the background of buffy coat smear when compared to the peripheral smear. The spatial and temporal distribution tends to be uneven in the buffy coat smear making the reliability of differential count doubtful as seen in figure 1 . This can be overcome to some extent by mixing appropriate volume of plasma with the buffy coat before making a smear on the clean glass slide [13]. But similar cells tend to crowd or aggregate in a buffy coat smear as confirmed by studies long back [16], which is the reason behind the clusters seen in the buffy coat smear.

\section{Conclusion}

The leukocyte distribution and morphological appearance is different in buffy coat smear when compared with traditional peripheral smears. Results from buffy coat are not reliable. There is significant numerical variation in number of cells such as neutrophils and lymphocytes in smears prepared from buffy coat which are comparatively more in blood circulation compared to cells like eosinophils and basophils. 


\section{Acknowledgement}

We are grateful to the Director and Principal of Medical Trust Institute of Medical Sciences, Medical Trust Hospital, Ernakulam, Kerala, India, for their support and timely help to conduct this study.

\section{Conflict of Interest}

There is no conflict of interest.

\section{Bibliography}

1. Carman H Robert. Hand Book of Medical Laboratory Technology. 2nd ed., Christian Medical Association of India (2016).

2. Gulati Gene., et al. "Purpose and Criteria for Blood Smear Scan, Blood Smear Examination, and Blood Smear Review". Annals of Laboratory Medicine 33.1 (2013).

3. Wenz B., et al. "The Clinical Utility of the Leukocyte Differential in Emergency Medicine". American Journal of Clinical Pathology 86.3 (1986): 298-303.

4. Chagas Carolina R. F., et al. "The Buffy Coat Method: A Tool for Detection of Blood Parasites without Staining Procedures". Parasites and Vectors 13.1 (2020).

5. Dou John., et al. "Cord Blood Buffy Coat DNA Methylation Is Comparable to Whole Cord Blood Methylation". Epigenetics 13.1 (2018).

6. Mondal Himel and Deepa P Budh. "Hematocrit (HCT)". StatPearls (2019).

7. Gajendra S., et al. "Leishman and Giemsa Stain: A New Reliable Staining Technique for Blood/Bone Marrow Smears". International Journal of Laboratory Hematology 37.6 (2015).

8. Blumenreich Martin S. "The White Blood Cell and Differential Count". Clinical Methods: The History, Physical, and Laboratory Examinations (1990).

9. Parente Jason. "Diagnostics for White Blood Cell Abnormalities: Leukocytosis and Leukopenia". Physician Assistant Clinics 4.3 (2019).

10. Teetson W., et al. "The Leukocyte Composition of Peripheral Blood Buffy Coat". American Journal of Clinical Pathology 79.4 (1983): 500-501.
11. Pflieger H., et al. "Differential Blood Counts from Cell Concentrates. A Comparison with Routine Differential Blood Counts". Acta Haematologica 61.3 (1979): 150-154.

12. Lantis Kay L., et al. "Elimination of Instrument-Driven Reflex Manual Differential Leukocyte Counts: Optimization of Manual Blood Smear Review Criteria in a High-Volume Automated Hematology Laboratory". American Journal of Clinical Pathology 119.5 (2003): 656-662.

13. Pereira Irma T., et al. "Preparation of Buffy Coat Smears in Leukopenic Patients". Laboratory Medicine 12.2 (1981): 96-98.

14. Longdoh Njunda Anna., et al. "Comparing the Buffy Coat and Traditional Blood Smears in the Microscopic Diagnosis of Malaria". International Journal of Malaria Research and Reviews 2.2 (2014).

15. Turhan Turan., et al. "Effects of Storage Conditions on Complete Blood Cell Count Parameters". Turkish Journal of Biochemistry 36.2 (2011): 165-174.

16. EFRATI P and L ROZENSZAJN. "The Morphology of Buffy Coat in Normal Human Adults”. Blood 16 (1960).

\section{Volume 5 Issue 10 October 2021 \\ (C) All rights are reserved by Deepa Revi., et al.}

